- https://doi.org/10.19048/2411-8729-2020-6-1-21-26

(c) BY 4.0

\title{
ОЦЕНКА ПСИХОЛОГИЧЕСКОЙ
}

И ЭМОЦИОНАЛЬНОЙ СФЕРЫ ПАЦИЕНТА

В ПРЕДУПРЕЖДЕНИИ КОНФЛИКТНЫХ СИТУАЦИЙ

ИЗ-ЗА ПРОФЕССИОНАЛЬНЫХ ОШИБОК,

ДЕФЕКТОВ ОКАЗАНИЯ СТОМАТОЛОГИЧЕСКОЙ

ПОМОЩИ И ОСОБЕННОСТЕЙ ЛИЧНОСТНОГО

РЕАГИРОВАНИЯ НА БОЛЕЗНЬ

А. К. Иорданишвили ${ }^{1,2, *}$ Е. Х. Баринов ${ }^{3}$, С. Ю. Тытюк ${ }^{2,4}$

${ }^{1}$ ФГБОУ ВО «Северо-Западный государственный медицинский университет им. И. И. Мечникова»

Министерства здравоохранения Российской Федерации,

Санкт-Петербург, Российская Федерация

${ }^{2}$ ФГБВОУ ВО «Военно-медицинская академия им. С. М. Кирова»

Министерства обороны Российской Федерации,

Санкт-Петербург, Российская Федерация

${ }^{3}$ ФГБОУ ВО «Московский государственный медико-стоматологический университет

им. А. И. Евдокимова» Министерства здравоохранения Российской Федерации,

Москва, Российская Федерация

4 ЧОУ ВО «Санкт-Петербургский медико-социальный институт»,

Санкт-Петербург, Российская Федерация

Аннотация. Актуальной в клинической стоматологии является профилактика возникновения конфликтных ситуаций.

Цель. С учетом внутренней картины болезни путем оценки психологической и эмоциональной сферы пациента предупреждать возникновение конфликтных ситуаций, возникающих в результате профессиональных ошибок, дефектов оказания стоматологической помощи.

Материал и методы. Оценка психологической и эмоциональной сферы с помощью синдрома психо-сенсорно-анатомо-функциональной дезадаптации, а также удовлетворенности стоматологической реабилитацией у 77 пациентов (36 мужчин и 41 женщина) в возрасте от 52 до 67 лет с использованием несъемных (1 группа), съемных (2 группа), зубных протезов на дентальных имплантатах (3 группа).

Результаты. Показана возможность выявления клинических случаев, в которых возможно возникновение конфликтных ситуаций из-за профессиональных ошибок, дефектов оказания стоматологической помощи или в связи особенностями личностного реагирования пациента на болезнь.

Заключение. Для профилактики жалоб и претензий пациентов в таких клинических ситуациях необходимо устранение дефектов оказания стоматологической помощи и (или) специальная психологическая подготовка таких пациентов до начала ортопедического стоматологического лечения.

Ключевые слова: судебно-медицинская экспертиза, дефекты оказания медицинской помощи, ненадлежащее оказание медицинской помощи, стоматологическая помощь, зубное протезирование, стоматологическая реабилитация, внутренняя картина болезни

Конфликт интересов: конфликт интересов отсутствует.

Финансирование: исследование не имело спонсорской поддержки.

Для цитирования: Иорданишвили А. К., Баринов Е. Х., Тытюк С. Ю. Оценка психологической и эмоциональной сферы пациента в предупреждении конфликтных ситуаций из-за профессиональных ошибок, дефектов оказания стоматологической помощи и особенностей личностного реагирования на болезнь. Судебная медиизина. 2020;6(1):21-26. https://doi.org/10.19048/2411-8729-2020-6-1-21-26

Поступила 27.02.2019

Принята после доработки 18.01.2020

Опубликована 28.03.2020 


\title{
ASSESSMENT OF THE PSYCHOLOGICAL AND EMOTIONAL STATE OF THE PATIENT FOR THE PREVENTION OF CONFLICT SITUATIONS ARISING FROM PROFESSIONAL ERRORS, INADEQUATE PROVISION OF DENTAL CARE AND PERSONAL RESPONSE TO THE DISEASE
}

\author{
Andrey K. Iordanishvili ${ }^{1,2,}$, Evgeny Kh. Barinov ${ }^{3}$, Sergey Yu. Tytyuk ${ }^{2,4}$ \\ ${ }^{1}$ North-Western State Medical University named after I. I. Mechnikov, \\ Saint Petersburg, Russian Federation \\ ${ }^{2}$ Military Medical Academy named after S. M. Kirov, \\ Saint Petersburg, Russian Federation \\ ${ }^{3}$ A. I. Yevdokimov Moscow State University of Medicine and Dentistry, \\ Moscow, Russian Federation \\ ${ }^{4}$ Saint-Petersburg Medico-Social Institute, \\ Saint Petersburg, Russian Federation
}

\begin{abstract}
Prevention of conflict situations is a relevant issue in clinical dentistry.
Aim. To prevent conflict situations that arise from professional errors and inadequate provision of dental care while considering the internal picture of the disease through the assessment of the psychological and emotional state of the patient.
\end{abstract}

Material and methods. We assessed the psychological and emotional state of 77 (36 men and 41 women) patients aged 52 to 67 years that had fixed (group 1), removable (group 2) dentures on dental implants (group 3) using the syndrome of psycho-sensory-anatomical and functional (PSAF) maladjustment, as well as considering their satisfaction with dental rehabilitation.

Results. The possibility of identifying clinical cases in which conflicts may arise due to professional errors, inadequate provision of dental care or due to the patient's personal response to the disease is shown.

Conclusion. In order to prevent patients' complaints in such clinical situations, it is necessary to ensure the quality dental care and (or) special mental preparation of such patients needs to be completed prior to denture treatment.

Keywords: forensic medical examination, poor quality of medical care, inadequate healthcare provision, dental care, denture, dental rehabilitation, internal picture of the disease

Conflict of interest: the authors declare no apparent or potential conflicts of interest.

Funding: the study had no sponsorship.

For citation: Iordanishvili A. K., Barinov Evgeny Kh., Tytyuk Sergey Yu. Assessment of the Psychological and Emotional State of the Patient for the Prevention of Conflict Situations Arising from Professional Errors, Inadequate Provision of Dental Care and Personal Response to the Disease. Russian Journal of Forensic Medicine. 2020;6(1):21-26. (In Russ.) https://doi.org/10.19048/2411-8729-2020-6-1-21-26

Submitted 27.02.2019

Revised 18.01.2020

Published 28.03.2020

В Российской Федерации медицина постепенно переходит в ряд «опасных» профессий, так как врач все чаще становится «жертвой» судебных исков со стороны пациентов, которых, по их мнению, лечили неправильно или небрежно [1-3]. Обращает на себя внимание, что на первое место вышли судебные дела в отношении врачей-стоматологов в сравнении с врачами других специальностей $[4,5,9]$. Определено, что 55 \% всех претензий к врачам-стоматологам возникает вследствие оказания ортопедической помощи, 33 \% - терапевтической, 12 \% случаев - после амбулаторного хирургического лечения $[6,10]$.

Описаны клинические наблюдения, когда при некачественном оказании зубопротезной помощи пациенты довольны результатами стоматологической реабилитации и не высказывают жалоб, претензий в адрес врачей-стоматологов, а также клинические случаи, когда при качественном оказании стоматологической реабилитации с использованием зубных протезов различных конструкций возникают конфликты между пациентом и лечебно-профилактическим учреждением, которые в подавляющем большинстве случаев завершаются в судебном порядке $[7,11]$.

Поэтому проблема «мирного» разрешения конфликтов в стоматологической практике является весьма актуальным звеном в становлении механизмов, которые, с одной стороны, обеспечивали бы продолжительный срок клинического благополучия пациента после стоматологической реабилитации и с другой - защищали бы врача от необоснованных претензий пациентов [8].

Нам представляется, что более важной для клинической стоматологии является профилактика возникновения конфликтных ситуаций. Понимая, что это возможно при нестандартном подходе, учитывающем психологию, эмоциональную сферу пациента, требующем дополнительных затрат времени врача, а следовательно, и материальных средств, мы попытались найти наиболее подходящую методику прогнозирования неудовлетворенности паци- 
ентов результатами стоматологической реабилитации. Этого удалось добиться путем внедрения в клиническую стоматологическую практику исследования синдрома психо-сенсорно-анатомо-функциональной дезадаптации и методики удовлетворенности пациентов результатами оказанной им стоматологической помощи.

Целью работы являлось путем оценки психологической и эмоциональной сферы пациента предупреждать возникновение конфликтных ситуаций, возникающих в результате профессиональных ошибок, дефектов оказания стоматологической помощи или в связи особенностями личностного реагирования пациента на болезнь, то есть с учетом внутренней картины болезни.

\section{$\checkmark$ МАТЕРИАЛ И МЕТОДЫ}

Для оценки психологической и эмоциональной сферы пациента, изучения особенностей личностного реагирования пациента на болезнь проведено комплексное обследование и стоматологическая реабилитация 77 пациентов (36 мужчин и 41 женщина) в возрасте от 52 до 67 лет с частичной или полной утратой естественных зубов на обеих челюстях. Пациенты проходили лечение в амбулаторно-поликлинических лечебно-профилактических учреждениях и стоматологических клиниках и были разделены на группы (рис. 1). У 27 чел. (1 группа) стоматологическая реабилитация была осуществлена с помощью различных конструкций несъемных зубных протезов (искусственные коронки и мостовидные протезы). Во 2 группе пациентов (31 чел.) протезирование было осуществлено частичными или полными съемными зубными протезами, в том числе в сочетании с коронками и мостовидными протезами. У лиц 3 группы (19 чел.) выполнена стоматологическая реабилитация различными зубными протезами, которые фиксировались на искусственных опорах (различные конструкции дентальных имплантатов).

Кроме общепринятой клинико-рентгенологической оценки при диагностике и выполнении стоматологической реабилитации пациентов осуществляли оценку личностного реагирования на утрату зубов и лечение (внутренняя картина болезни) с использованием синдрома психо-сенсорно-анатомо-функциональной дезадаптации (СПСАФД). Согласно этой методике все проявления внутренней картины заболевания распределяются по 4 кластерам: «П» - психологический (психический), «С»- сенсорный, «А» - анатомический и «Ф» - функциональный. Особенностью СПСАФД является то, что оценку выраженности отдельных симптомов, ощущений, вызывающих дезадаптацию, дают сами пациенты.

Для этого использовали единую аналогово-балльную шкалу самооценки пациентами выраженности отдельных проявлений патологии (от 1 до 9 баллов: от «не беспокоит» (1 балл), до «слабо» (3 балла), «умеренно» (5 баллов), «сильно» (7 баллов) и «чрезвычайно сильно» (9 баллов) беспокоит). Распределение отдельных симптомов, вызывающих у пациентов дезадаптацию, осуществлял врач-стоматолог. Результаты самооценки пациентами выраженности проявления стоматологической патологии осуществляли путем учета интегрального показатели выраженности СПСАФД (показатель S, рассчитывался в баллах), а также с учетом структуры интегрального показателя СПСАФД согласно кластерам («П», «С», «А», «Ф», рассчитывался в баллах).

Для интегральной оценки выраженности (напряженности) внутренней картины болезни использовали градацию интегрального показатели выраженности СПСАФД - показателя S. При значении этого показателя от 4 до 9,9 усл. ед. считали, что пациент, несмотря на наличие стоматологической патологии, адаптирован к условиям существования. При значениях показателя S, равных 10,0-16,9 усл. ед., считали, что у пациента изза имеющейся стоматологической патологии нарушена приспособляемость к условиям существования. При значениях показателя S, равных 17,0-36,0 усл. ед., считали, что у пациента, из-за стоматологической патологии имеется состояние дезадаптации. Оценку внутренней картины заболевания у обследуемых осуществляли дважды: до начала стоматологического лечения и в сроки от 14 до 30 суток после завершения стоматологической реабилитации.

Для оценки удовлетворенности пациентов стоматологической реабилитацией была также использована единая аналогово-балльная шкала самооценки пациентами удовлетворенности стоматологической реабилитацией. При оценке удовлетворенности считали, что при значении 1 - пациенты не удовлетворены лечением; 3 - удовлетворены лечением слабо; 5 - умеренно удовлетворены лечением; 6 - удовлетворены лечением частично; 8 полностью удовлетворены лечением; 9 - чрезвычайно удовлетворены лечением. Удовлетворенность оценивали однократно, в период с 14 по 30 сутки после завершения стоматологической реабилитации, одновременно с определением динамики внутренней картины болезни (личностного реагирования) на стоматологическое лечение. Особенностью СПСАФД и методики оценки удовлетворенности является то, что оценку выраженности отдельных симптомов, ощущений, вызывающих дезадаптацию, дают сами пациенты.

Достоверность различий средних величин независимых выборок подвергали оценке при помощи параметрического критерия Стьюдента при нормальном законе распределения и непараметрического критерия Манна Уитни при отличии от нормального распределения показателей. Проверку на нормальность распределения оценивали при помощи критерия Шапиро - Уилкса. Для статистического сравнения долей с оценкой достоверно-

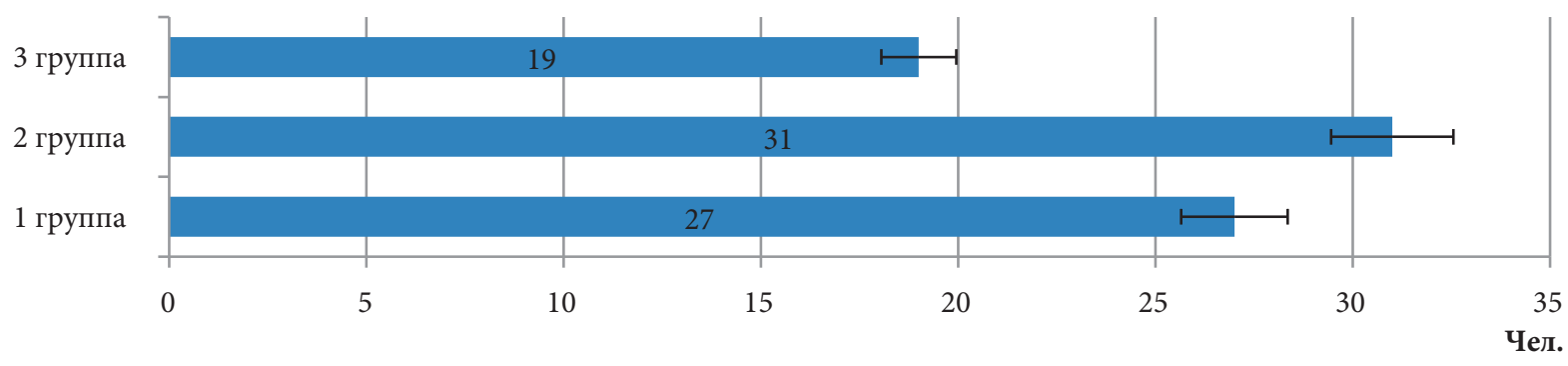

Рис. 1. Распределение пациентов на группь исследования (чел.)

Fig. 1. Distribution of patients into study groups (people) 
сти различий применяли критерий Пирсона $\chi^{2}$ с учетом поправки Мантеля - Хэнзеля на правдоподобие. Во всех процедурах статистического анализа считали достигнутый уровень значимости (р), критический уровень значимости при этом был равным 0,05.

\section{$\checkmark$ РЕЗУЛЬТАТЫ ИССЛЕДОВАНИЯ И ОБСУЖДЕНИЕ}

В ходе изучения показателей СПСАФД, как интегральных, так и структуры интегрального показателя S, определяющего внутреннюю картину болезни, достоверных половых различий не получено на протяжении всего исследования ( $p \geq 0,05)$. Поэтому статистическая обработка цифровых данных в ходе работы выполнена без учета пола. В ходе работы установлено, что до начала стоматологической реабилитации, независимо от группы пациентов, достоверных различий как по кластерам, так и по интегральному показателю S (рис. 2) не отмечено $(\mathrm{p} \leq 0,05)$.

Наименьшие значения были в кластере «С», определяющем сенсорные нарушения, которые составили 1,79-1,84 балла, а также в кластере «П» (2,75-2,93 балла), характеризующем психологическое состояние пациента. Наибольшие цифровые значения СПСАФД у пациентов всех групп исследования были отмечены в кластере «А»
(7,43-8,16 балла), характеризующем утрату зубов, а также в кластере «Ф» (5,67-6,12 балла), характеризующем главным образом нарушение функции жевания (рис. 3). Очевидно, утрата зубов в большей степени волновала пациентов в связи с имеющимися в полости рта дефектами зубных рядов или полной потерей зубов, а также из-за нарушения функции жевания.

По завершении стоматологической реабилитации получены достоверные различия как по значению интегрального показателя S в группах исследования, так и по цифровым значениям СПСАФД в кластерах «А» и «Ф» (рис. 2, 3). Так, если у пациентов 1 и 3 групп не выявлено достоверных различий по цифровым значениям СПСАФД во всех изученных кластерах и по интегральному показателю $\mathrm{S}$ ( $\mathrm{p} \geq 0,05)$, то изученные показатели СПСАФД после завершения стоматологической реабилитации, а именно интегральный показатель S и значения СПСАФД в кластерах «A» и «Ф» достоверно отличались от полученных после завершения лечения по сравнению с пациентами 2 группы исследования ( $\mathrm{p} \leq 0,05)$, которые пользовались частичными и (или) полными съемными зубными протезами. У пациентов 2 группы исследования интегральный показатель S, а также значения СПСАФД в кластерах «A» и «Ф» были практически в 2 раза выше, чем у лиц 1

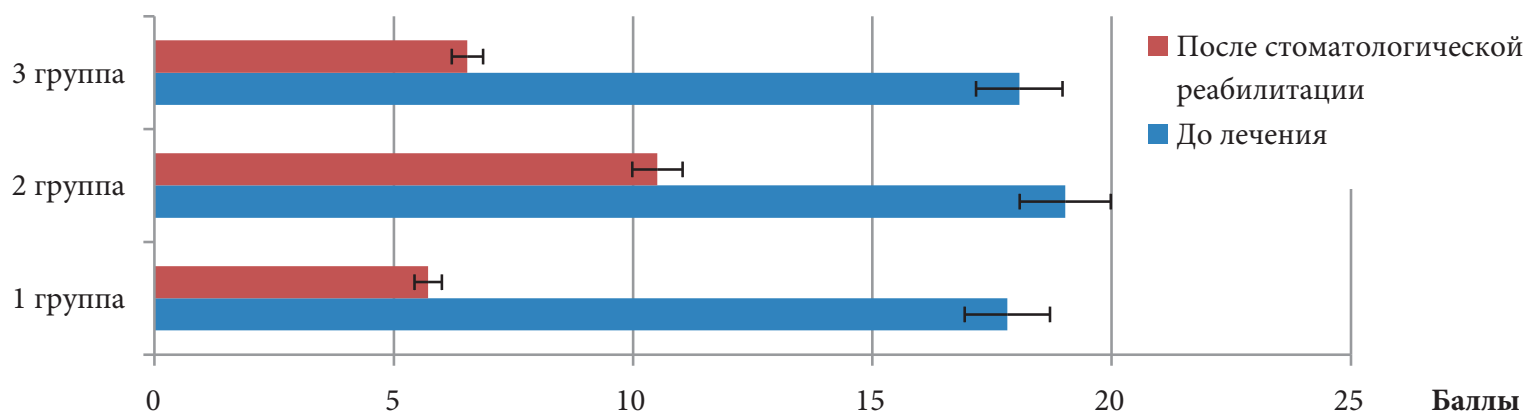

Рис. 2. Значения интегрального показателя $S$ у пациентов исследованных групп до и после завершения стоматологической реабилитации (балль)

Fig. 2. Integral indicator $S$ in patients of the studied groups prior to and following dental rehabilitation (points)

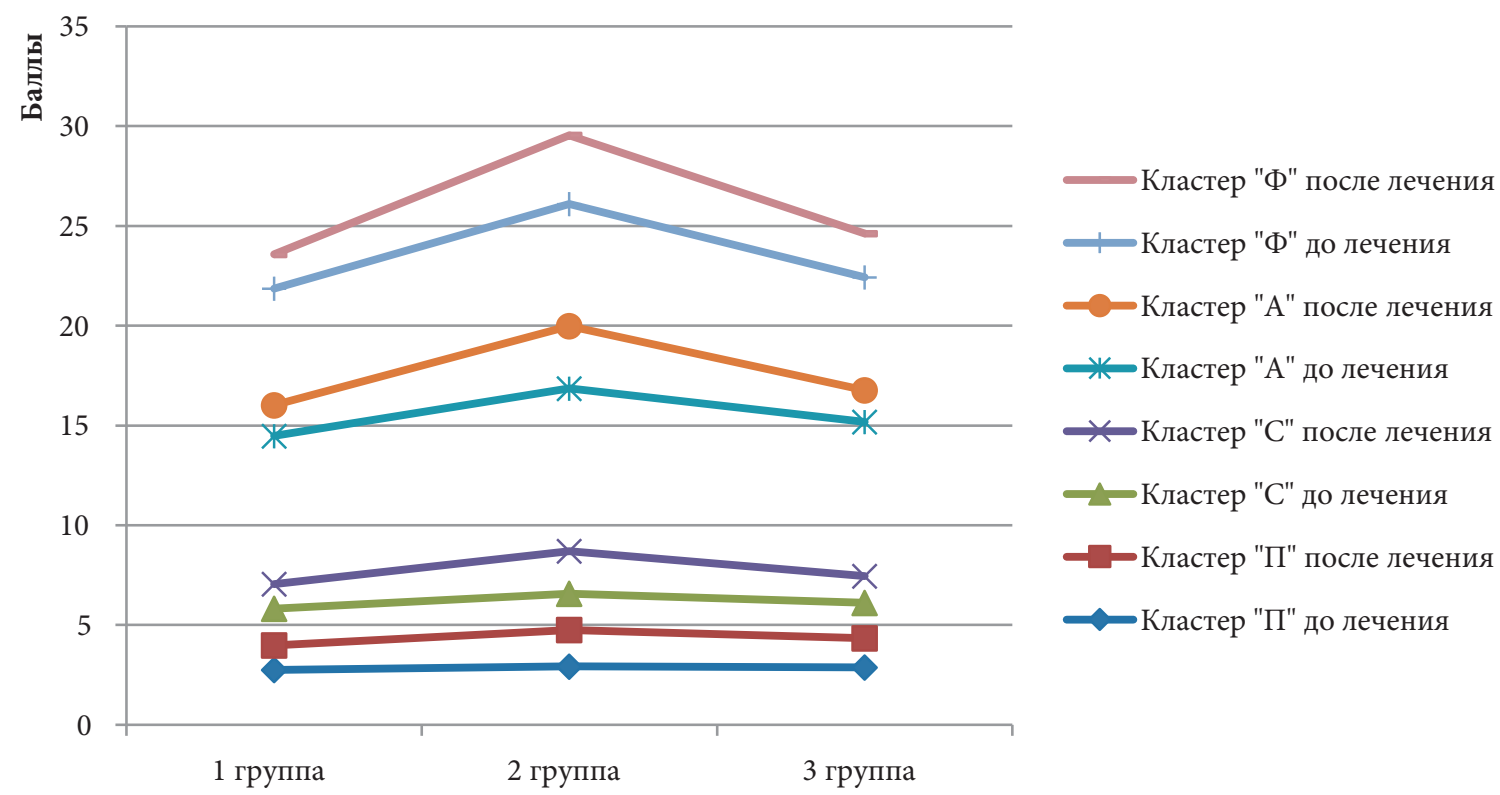

Рис. 3. Значения СПСАФД в исследуемых кластерах у обследованных пациентов разных групп (балль)

Fig. 3. Studied clusters of PSAF maladjustment syndrome in the examined patients from different groups (points) 


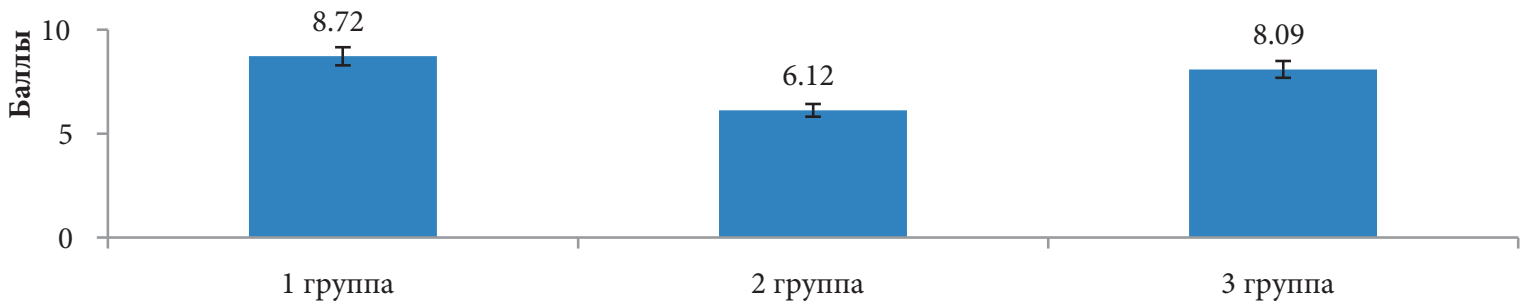

Рис. 4. Показатели удовлетворенности пациентов в группах исследования после завериения стоматологической реабилитации (балль)

Fig. 4. Patient satisfaction indicators in the study groups following dental rehabilitation (points)

и 3 групп, что, очевидно, обусловливалось конструкционными особенностями съемных зубных протезов.

Средние показатели удовлетворенности пациентов по группам исследования представлены на рисунке 4. По средним показателям в изучаемых группах пациентов лица 1 и 3 группы исследования были полностью удовлетворены стоматологической реабилитацией (показатель удовлетворенности составил 8,09-8,72 балла), а лица 2 группы удовлетворены стоматологической реабилитацией лишь частично (показатель удовлетворенности 6,12 балла). В то же время, анализируя анкеты, характеризующие удовлетворенность пациентов стоматологической реабилитацией индивидуально, установили, что удовлетворены лечением слабо или умеренно в 1, 2 и 3 группах соответственно 2, 5 и 2 человека. Анализ анкет, характеризующих СПСАФД, показал, что после завершения стоматологической реабилитации пациентов значения интегрального показателя $\mathrm{S}$ по сравнению со средними значениями в группе были на 25-30 \% выше, как правило, за счет равномерного увеличения значений во всех исследованных кластерах. При этом у всех 9 пациентов из исследованных групп, которые в разной степени не были удовлетворены стоматологической реабилитацией, выявлена достоверная зависимость повышения показателей в кластерах «П» и «С» $(\mathrm{p} \leq 0,05)$, а также интегрального показателя $\mathrm{S}(\mathrm{p} \leq 0,01)$ до начала стоматологической реабилитации на 23-35 \% по сравнению со средними значениями в исследуемой группе пациентов.

\section{$\checkmark$ ЗАКЛЮЧЕНИЕ}

Резюмируя вышеизложенное, можно заключить, что результаты проведенного клинического исследования показали возможность выявления клинических случаев, в которых возможно возникновение конфликтных ситуаций из-за профессиональных ошибок, дефектов оказания стоматологической помощи или в связи особенностями личностного реагирования пациента на болезнь. Клиническая стоматологическая практика показала эффективность использования синдрома психо-сенсорно-анатомо-функциональной дезадаптации для прогнозирования результатов стоматологической реабилитации пациентов. Установлено, что если у пациента перед началом стоматологического ортопедического лечения независимо от характера утраты зубов и планируемых конструкций зубных протезов для их замещения имеются повышенные показатели СПСАФД в кластерах «П» и «С» (выше 3-4 баллов), что объективно характеризует неблагоприятные особенности их личностного реагирования на болезнь, то это может являться прогностическим фактором возможных нарушений в адаптационном периоде после стоматологической реабилитации, для профилактики которых необходима разработка методики специальной психологической подготовки таких пациентов до начала ортопедического стоматологического лечения, что является задачей нашего дальнейшего исследования. $\checkmark$ ЛИТЕРАTУPA / REFERENCES

1. Иорданишвили А. К., Толмачев И. А., Сагалатый А. М. Анализ причин удаления зубов и качество заполнения медицинской документации в хирургическом кабинете стоматологической поликлиники. Институт стоматологии. 2008;3(40):30-31. [Iordanishvili A. K., Tolmachev I. A., Sagalatyi A. M. Analysis of reasons for tooth extraction and quality of filling out medical records in surgery of a dental clinic Institut stomatologii. 2008;3(40):28-29. (In Russ.)]

2. Иорданишвили А. К., Толмачев И. А., Бобунов Д. Н. Алгоритм судебно-медицинской экспертизы при оказании стоматологического ортопедического лечения. Институт стоматологии. 2009;1(42):10-12. [Iordanishvili A. K., Tolmachev I. A., Bobunov D. N. Forensic medical examination algorithm for dental orthopedic treatment. Institut stomatologii. 2009;1(42):10-12. (In Russ.)]

3. Иорданишвили А. К., Толмачев И. А., Альшевский В. В., Поленс А. А., Сериков А. А. Клиникоэкспертные аспекты амбулаторной диагностики заболеваний височно-нижнечелюстного сустава. Судебно-медииинская экспертиза. 2011;3:18-22. [Iordanishvili A. K., Tolmachev I. A., Al'shevskii V. V., Polens A. A., Serikov A. A. Clinico-expert aspects of diagnostics of temporomandibular joint disorders in out-patient medical and preventive treatment facilities. Sudebno-meditsinskaya ekspertiza. 2011;5:18-22. (In Russ.)]

4. Иорданишвили А. К., Веретенко Е. А., Мироненко А. Н. Медицинские, социальные, экономические и юридические аспекты стоматологического лечения людей старших возрастных групп. Экология и развитие общества. 2015;3(14):63-65. [Iordanishvili A. K., Veretenko E. A., Mironenko A. N. Medical, social, economic and legal aspects of stomatologic treatment of people of the senior age groups. Ekologiya $i$ razvitie obshchestva. 2015;3(14):63-65. (In Russ.)]

5. Иорданишвили А. К., Кувшинова А. К., Музыкин М. И., Сериков А. А. Сравнительная оценка методов стоматологической реабилитации при последствиях огнестрельных ранений челюстнолицевой области. Военно-медицинский журнал. 2018;339(9):43-47. [Iordanishvili A. K., Kuvshinova A. K., Muzykin M. I., Serikov A. A. Comparative evaluation of methods of dental rehabilitation with consequences of gunshot wounds in the maxillofacial area. Voenno-meditsinskii zhurnal. 2018;339(9):43-47. (In Russ.)]

6. Иорданишвили А. К., Веретенко Е. А., Солдатова Л. Н. Влияние метода фиксации полных съемных протезов на эффективность пользования и психофизиологический статус людей пожилого и старческого возраста. Институт стоматологии. 2014;4(65):28- 
34. [Iordanishvili A. K., Veretenko E. A., Soldatova L. N. Influence of fixation technique of total removable prostheses on the efficient use and the psycho-physiological status of senior and senile aged people. Institut stomatologii. 2014;4(65):28-34. (In Russ.)]

7. Иорданишвили А. К. Психическое здоровье протезоносителей при пользовании съемными зубными протезами. Клиническая стоматология. 2018;1(85):64-67. [Iordanishvili A. K. Mental health when using removable dentures. Klinicheskaya stomatologiya. 2018;1(85):64-67. (In Russ.)]

8. Иорданишвили А. К. Психическое здоровье пожилых людей при пользовании полными съемными зубными протезами: миф или реальность? Стоматология. 2018;5(96):56-61. [Iordanishvili A. K. Mental health of elderly and old people when using a complete dry dental prosthesis: myth or reality? Stomatologiya. 2018;5(96):56-61. (In Russ.)]

9. Felicetti D. M., Julliard K. Behaviours of children with and without attention deficit hyperactivity disorder during a dental recall visit. ASDC J. Dent Child. 2000;4(67):246-249.

10. Ismail A. I., Sohn W. The impact of universal access to dental care on disparities in caries experience in children. J. Am Dent Assoc. 2001;3(132):295-303.

11. Kreider K. A., Stratmann R. G., Milano M., Agostini F. G., Munsell M. Reducing children's injection pain: lidocaine patches versus topical benzocainei gel. Pediatr. Dent. 2001;1(23):19.

Об авторах • Authors

ИОРДАНИШВИЛИ Андрей Константинович - д.м.н., профессор, профессор кафедры ортопедической стоматологии ФГБОУ ВО «СЗГМУ им. И. И. Мечникова» Минздрава России; профессор кафедры челюстно-лицевой хирургии и хирургической стоматологии ФГБВОУ ВО «ВМА им. С. М. Кирова» Минобороны России [Andrey К. Iordanishvili, Dr. Sci. (Med.), Prof., Department of Prosthetic Dentistry, North-Western State Medical University named after I. I. Mechnikov; Prof., Department of Oral and Maxillofacial Surgery, Military Medical Academy named after S. M. Kirov] • professoraki@mail.ru•\{ORCID:0000-0003-0052-3277\}

БАРИНОВ Евгений Христофорович - д.м.н., профессор кафедры судебной медицины и медицинского права ФГБОУ ВО «МГМСУ им. А. И. Евдокимова» Минздрава России; профессор кафедры судебной медицины и медицинского права ФГАОУ ВО «РУДН» [Evgeny Kh. Barinov, Dr. Sci. (Med.), Prof., Department of Forensic Medicine and Medical Law, A. I. Yevdokimov Moscow State University of Medicine and Dentistry; Prof., Department of Forensic Medicine and Medical Law, Peoples' Friendship University of Russia]• ev.barinov@mail.ru

ТЫТЮК Сергей Юрьевич - к.м.н., ассистент кафедры челюстно-лицевой хирургии и хирургической стоматологии ФГБВОУ ВО «ВМА им. С. М. Кирова» Минобороны России [Sergey Yu. Tytyuk, Cand. Sci. (Med.), Research Assistant, Department of Oral and Maxillofacial Surgery, Military Medical Academy named after S. M. Kirov] •tmos2005@ yandex.ru

- Вклад авторов. Авторы несут полную ответственность за предоставление окончательной версии рукописи в печать. Все авторы принимали участие в разработке концепции статьи и написании рукописи. Окончательная версия рукописи была одобрена всеми авторами. Авторы благодарны анонимным рецензентам за полезные замечания.

- Contributions. Authors are solely responsible for submitting the final manuscript to print. All authors participated in the development of the concept of the article and the writing of the manuscript. The final version of the manuscript was approved by all authors. The authors are grateful to anonymous reviewers for helpful comments. 